\title{
EDITORIAL
}

\section{GreenLight photoselective vaporisation of the prostate}

Over the past two decades, treatment options for benign prostatic hyperplasia (BPH) have undergone several dramatic changes. Up until the late 1980's, management options were confined to either watchful waiting or transurethral resection of the prostate (TURP). During the 1990's, two classes of medical therapy became available: the $\alpha$-blockers and the $5 \alpha$-reductase inhibitors. Gradually, internationally, the number of TURPs fell, as more and more patients opted for medical therapy rather than surgery. From that time on, and right up to the present, urologists have been searching for an interventional therapy with less morbidity than TURP and greater efficacy than medical therapy. Balloon dilatation and microwave therapy, as well as higher powered thermotherapy, came and went, as did the first generation side-firing laser devices.

Judging from the data included in the articles published in this supplement of Prostate Cancer and Prostatic Diseases, the advent of photoselective vaporization of the prostate (PVP) may have provided for the first time a truly effective minimally invasive therapy with the requisite low morbidity. Patients are understandably reluctant to take medical therapy for $\mathrm{BPH}$ indefinitely, and are also worried about the complications of TURP, especially bleeding and incontinence. They also are keen to return to their normal activities of daily living as soon as possible. The GreenLight PVP now offers them the opportunity of an almost bloodless day case procedure with an almost zero incidence of incontinence. The device carries particular advantages over traditional TURP in patients who are treated with anticoagulants such as warfarin, clopidogrel or aspirin. All patients should be warned, however, that some dysuria may occur and that some minor secondary bleeding can occur 7-10 days after the procedure. The new higher powered GreenLight laser, described by Gordon Muir and Alexis $\mathrm{Te}$ in their articles, may reduce the time required to complete the operation and this may be especially helpful in patients with a large prostate where more energy is usually required.

More work needs to be done to confirm the durability of treatment effect and to confirm the cost effectiveness of the PVP compared with TURP or long-term medical therapy, but on the balance of available evidence, this new addition to the urologists armamentarium seems a most attractive treatment option.

RS Kirby

Co-Editor

Prostate Cancer and Prostatic Disease 